\title{
Qualitative Analysis of dual MAC in Multihop Adhoc Wireless Network
} doi : https://doi.org/10.32628/CSEIT217235

\section{Amit Kumar \\ Department of Computer Science and Engineering, Maulana Azad College of Engineering and Technology, Patna, Bihar, India}

\section{Article Info}

Volume 7, Issue 2

Page Number: 128-136

Publication Issue :

March-April-2021

\section{Article History}

Accepted : 25 March 2021

Published : 31 March 2021

\section{ABSTRACT}

Multihop adhoc wireless networks offer great challenges for protocol designers. Stations in such networks are constrained by factors like low power, limited bandwidth, link errors and collisions. Changes are needed at various levels of the protocol stack, most importantly at the media access layer(MAC). This paper emphasizes in minimizing the collisions, and take care of the hidden and exposed problems in multihop wireless networks. The MAC with Distributed Coordination Function(DCF)does not scale well in such networks. We introduce Point Coordination Function(PCF)in the region of high traffic areas, and discuss its effect on network performance. To improve network scalability and throughput, we propose the design of dual MAC. This work discusses architecture and working of the dual MAC. Performance results of the network using MAC are presented, and compared with that of pure DCF operation.

Keywords: Distributed Coordination Function, Media Access Layer, Multihop Wireless Networks, Point Coordination Function

\section{INTRODUCTION}

In recent times, the wireless networks have become very popular. Wireless LANs are being deployed on airports, conferences, etc. People have started using portable laptops to access Internet and other resources using wireless networks while moving. Another area which has generated a lot of interest recently, is wireless ad-hoc networks. An ad-hoc network is formed when two or more stations come together to form an independent network. Ad-hoc networks are also termed as infrastructure-less networks since as they do not require any prior infrastructure. Two stations that are within transmission range of each other are called one hop neighbours. Multihop ad-hoc networks are ones in which the stations can talk to stations more than one hop away via intermediate stations. Cooperative adhoc networks are formed by several homogeneous wireless stations. All the stations cooperate with each other, i. e. , the traffic for the stations that are more than one hop away is routed by the intermediate stations. The intermediate stations are called relaying stations.

Deployed in 1990's, Mobile Ad-hoc networks have been Networks are a collection of two or more devices equipped with wireless communications and 
networking capability. These devices can communication with other nodes that immediately within their radio range or one that is outside their radio range. For the later, the nodes should deploy an intermediate node to be the router to route the packet from the source toward the destination. The Wireless Ad-hoc Networks do not have gateway, every node can act as the gateway. Although since 1990s', lots of research has been done on this particular field, ithas often been questioned as to whether the architecture of Mobile Ad-hoc Networks is a fundamental flawed architecture. The main reason for the argument is that Mobile Ad-hoc Networks are almost never used in practice, almost every wireless network nodes communicate to base-station and access points instead of co-operating to forward packets hop-by-hop.

\section{Mobile Ad-hoc Networks(MANET) are a} fundamentally flawed architecture. As argument, we try to clarify the definition, architecture and the characters of MANET, as well as the main challenges of constructing the MANET. Although many works have been done to solve the problem, we will show in this paper that it is very difficult to solve these limitations which made the Mobile Ad-hoc Networks a flawed architecture. After giving many evidences and analysis, we could see that the key technologies of Wireless Ad-hoc Networks were not implemented as well as we expect. That is to say many problems are inherently unsolvable. Thus, we could explain why we take the position that Mobile Ad-hoc Networks are flawed architecture network are distributed among the terminals themselves. The entire network is mobile, and the individual terminals are allowed to move freely. In this type of networks, some pairs of terminals may not be able to communicate directly with each other and have to relay on some terminals so that the messages are delivered to their destinations. Such networks are often refereed to as multi-hop or store-and- forward networks. The nodes of these networks function as routers, which discover and maintain routes to other nodes in the networks. The nodes may be located in or on airplanes, ships, trucks, cars, perhaps even on people or very small devices. Mobile Ad-hoc Networks are supposed to be used for disaster recovery, battlefield communications, and rescue operations when the wired network is not available. It can provide a feasible means for ground communications and information access.

\section{Media Access Control}

In the seven-layer OSI model of computer networking, media access control (MAC) data communication protocol is a sublayer of the data link layer, which itself is layer 2. The MAC sublayer provides addressing and channel access control mechanisms that make it possible for several terminals or network nodes to communicate within a multiple access network that incorporates a shared medium, e. g. Ethernet. The hardware that implements the MAC is referred to as a medium access controller. The link characteristics in wireless environments is very different from that of wired networks. At link layer we have to face following challenges:

Bandwidth : Bandwidth is the one of the most scarce resource in wireless networks. The available bandwidth in wireless networks $(2-10 \mathrm{Mbps})$ is far less than the wired links (typically $100 \mathrm{Mbps}$ ). Range Issues: The transmission range of stations depends upon the transmitted power and various sensitivity values. Unlike wired networks all stations on a LAN can not listen to one another.

Power : The wireless stations are battery operated and therefore higher transmission power leads to faster degeneration of the batteries. On the other hand, if we keep transmission power too small, the stations may no longer be in range of each other. 
Collisions : Since all stations can not listen to each other, transmission from two station may lead to collision at another station.

Link Errors : Channel fading and interference cause link errors and these errors may sometimes be very severe.

The transmission range of stations in wireless network is limited by the transmission power, therefore, all the station in a LAN can not listen to each other. This means that normal carrier sense mechanism which assumes that all stations can listen to each other, fails. In particular, this gives rise to hidden node and exposed node problem. Consider stations A, B, C and D. Hidden and exposed node problem can be described as follows :

Hidden node Problem: Stations $\mathrm{A}$ and $\mathrm{C}$ become hidden to each other as station $\mathrm{B}$ can listen both to $\mathrm{A}$ and $\mathrm{C}$, but stations $\mathrm{A}$ and $\mathrm{C}$ can not listen to each other. If

a packet is being transmitted from station $\mathrm{A}$ to station $\mathrm{B}$ and station $\mathrm{C}$ decides to

start a transmission (being hidden, it does not know of A-B transmission), there

will be collision at station $B$.

Exposed node Problem: If station B is sending data to station $\mathrm{A}$, then station $\mathrm{C}$

becomes exposed to $\mathrm{B}$ and is forced to be silent even if it can send data to another

station. This is because station $\mathrm{C}$ finds carrier busy during transmission of station $\mathrm{B}$.

A simple and elegant solution to the hidden node problem is to use small packets called RTS (Request to Send) and CTS (Clear to Send) for handshaking before transmission of data packet.

Point coordination function (PCF) is a Media Access Control (MAC) technique used in IEEE 802. 11 based WLAN. It resides in a point coordinator also known as Access Point (AP), to coordinate the communication within the network. The PCF is located directly above the Distributed Coordination Function (DCF), in the IEEE 802. 11 MAC Architecture. Channel access in PCF mode is centralized and hence the point coordinator sends CF-Poll frame to the PCF capable station to permit it to transmit a frame. In case the polled station does not have any frames to send, then it must transmit null frame. Due to the priority of PCF over DCF, stations that only use DCF might not gain access to the medium. To prevent this, a repetition interval has been designed to cover both (Contention free)PCF \& (Contention Based)DCF traffic. The repetition interval which is repeated continuously, starts with a special control frame called Beacon Frame. When station hear the beacon frame, they start their network allocation vector for the duration of the contention free period of the repetition period.

The 802. 11 MAC offers contention free service by means of PCF. PCF is optional capability and provides contention-free $(\mathrm{CF})$ frame transfers. The PCF relies on the point coordinator (PC) to poll other stations. The polled stations can send the data without contending for the medium. In a wireless LAN, the function of a PC is performed by AP within each BSS.

The PCF is required to coexist with the DCF and logically sits on the top of DCF . The PCF consists of alternating contention free period (CFP), and contention period (CP) . In the CFP, the PC polls each of the stations present in the BSS. The PC specifies the start of the CFP by sending a beacon that contains the length of CFP duration, among other things. All the stations in the BSS set their NAV for the duration of the CFP. The PC terminates the CFP by sending a CF-End frame, and may also terminate it before the advertised CFP duration. The time difference between two beacons is called beacon 
period (BP) or CFP repetition interval, and is a multiple of beacon frame. The beacon also helps in synchronization and timing. The limits on durations of each of the frames are described in the IEEE 802. 11 standard . In the CP, the stations use DCF to access the medium.

In CFP, the PC polls each station by sending either Poll, Data + Poll or Data + ACK (for previous frames) + Poll. The polled station sends the data or Data + ACK (if polled by Data+Poll frame) to PC in response to the PC's poll. It is also possible to have station to station transmission during the CFP. The PCF offers connection oriented polled service and therefore it reduces contention to a large extent. It is ideal for wireless LAN environments where all the stations are connected to the AP over a single hop. However, at low traffic the overheads are quit high.

Distributed coordination function (DCF) is the fundamental MAC technique of the IEEE 802. 11 based WLAN standard. DCF employs a CSMA/CA with binary exponential backoff algorithm.

DCF requires a station wishing to transmit to listen for the channel status for a DIFS interval. If the channel is found busy during the DIFS interval, the station defers its transmission. In a network where a number of stations contend for the wireless medium, if multiple stations sense the channel busy and defer their access, they will also virtually simultaneously find that the channel is released and then try to seize the channel. As a result, collisions may occur. In order to avoid such collisions, DCF also specifies random backoff, which forces a station to defer its access to the channel for an extra period. DCF also has an optional virtual carrier sense mechanism that exchanges short Request-to-send (RTS) and Clear-tosend (CTS) frames between source and destination stations during the intervals between the data frame transmissions. DCF includes a positive acknowledge scheme, which means that if a frame is successfully received by the destination it is addressed to, the destination needs to send an ACK frame to notify the source of the successful reception. DCF does not solve the hidden terminal and/or exposed terminal problem completely, it only alleviates the problem through the use of RTS and CTS, and recommends the use of a larger carrier sensing range. DCF is defined in subclause 9. 2 of the IEEE 802. 11 standard and is the de facto default setting for Wi-Fi hardware. 802. 11 DCF consumes a significant amount of airtime, 802. 11 control messages usually convey very little information. For example, an ACK message can take up to $60 \mu \mathrm{s}$ to transmit completely, which includes an amount of airtime sufficient to transmit 3240 bits at $54 \mathrm{Mbit} / \mathrm{s}$, during which it conveys a single bit of relevant information. The IEEE 802. 11 standard also defines an optional access method using a Point Coordination Function (PCF). PCF allows the access point acting as the network coordinator to manage channel access. The IEEE 802. 11e amendment to the standard enhances the DCF and the PCF, through a new coordination function called Hybrid Coordination Function (HCF).

Simple DCF is not suitable for centralized multihop network due to collisions at high traffic. A polling MAC (PCF) is required at the center of the network to handle high traffic and reduce collision, but it gives rise to hidden and exposed node problems. The solution to both of these problems is provided by introducing dual Nodes at the boundary of the central node.

\section{Need For dual MAC}

The centralized multihop ad hoc networks, as explained in earlier chapters, require a MAC that can provide distributed access mechanism. Furthermore, the MAC should be able to handle high traffic in central region. The pure DCF operation, or hybrid 
operation do not perform efficiently in multihop networks. The reasons for this inefficiency are:

1. The DCF does not work well in high load scenario.

2. In case of hybrid operation, the polling and NAV setting in PCF nodes cause exposed and hidden node problems, thereby decrease the throughput.

To improve the throughput, boundary nodes should be able to receive date from outer nodes during the $\mathrm{CFP}$ period (NAV is set). For this the MAC should be able to receive even if its NAV is set. Also, transmissions from outer stations should not collide with that of PC at boundary stations. To address above problems, we propose to equip boundary stations with dual MAC.

\section{Architecture of Dual MAC}

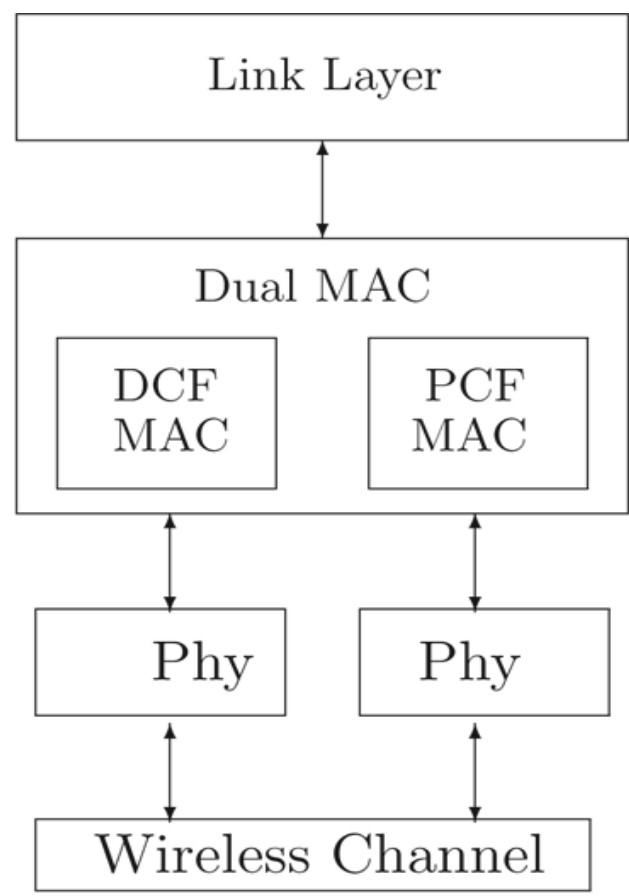

Fig. 1: Architecture of Dual MAC

The dual MAC consist of two MACs in a single station each capable to send and receive packets independently. Each MAC is designed to operate on different logical channels. As mentioned earlier, the channels can be either FDMA or CDMA but it does not make difference as far as the design of the dual MAC is concerned. As shown in Figure 5. 1, the dual MAC is below the Link Layer and basically encapsulate two actual 802. 11 MACs. These two MACs which we refer to as PCF MAC and DCF MAC respectively, communicate on two different logical channels. The DCF MAC talks to the stations that are operating in DCF mode and the PCF MAC talks to the PC and other stations which are in PCF mode. The dual MAC is a layer on the top of these MACs and single point of interface to the Link Layer (LL). For all down-going packets the dual MAC layer acts as arbitrator and sends on either of the MAC below it. For the up-going packets, the job of dual MAC is simply to hand over the packet to the link layer.

\section{Operation}

A packet arriving from link layer is received by the dual MAC and handed over to the MAC at appropriate frequency. The link layer finds out the MAC address of the next hop destination by using ARP and hands out the packet to the dual MAC layer along with the destination MAC address. In case dual MAC, the dual MAC also needs to know the channel of the destination station. This could be done either by ARP table maintaining information about the channel on which the destination stations is communication, or by maintaining a local list of stations on each channel. The dual MAC figures out the channel of the destination MAC and sends out the packet to the appropriate MAC. The broadcast packets like route discovery packets and ARP packets are sent to both the MACs. On receiving a packets from lower layer, the dual MAC simply hands it out to the link layer. The dual MAC is essentially a MAC which can access two separate logical channels. This can be done in either half duplex way or in full duplex way. In half duplex method, the logical 
channel (radio frequency in case of FDMA, PN code in case of CDMA) is changed by the radio for a different channel to be accessed. In full duplex method, both the channel are accessed simultaneously. This requires that two different radios be employed for full duplex operation. We choose the full duplex operation since it offers parallelism of channel access which is essential for the boundary nodes. This requires extra cost, but the addition in cost is justified considering that very few stations need to be equipped with the dual MAC.

\section{Node Architecture and implementation in NS}

The dual Node differs from the architecture of the original NS node object only at the MAC layer. The MAC layer consists of a dual MAC object which encapsulates two 802. 11 MAC objects within IT. The dual Node differs from the architecture of the original NS node object only at the MAC layer. The MAC layer consists of a dual MAC object which encapsulates two 802. 11 MAC objects within it. All the upcoming and downgoing packets are first received by the dual MAC. In case of down going packets, the dual MAC determines the channel on which this packet is to be sent, and handles it over to appropriate MAC. In case of ingoing packets, it simply hands over the packets to the MAC corresponding to received channel where it is handed to link layer after processing.

The dual MAC (class Mac Dual) is implemented by deriving the existing Mac class in NS. The Mac Dual object encapsulates two actual Mac 80211 objects. The Mac 80211 class and Packet class have also changed to incorporate dual MAC functionality.

The dual MAC (class Mac Dual) is implemented by deriving the existing Mac class in NS. The Mac Dual object encapsulates two actual Mac 80211 objects. The Mac 80211 class and Packet class have also changed to incorporate dual MAC functionality. The
Mac class has been change to incorporate the features necessary for the dual MAC. The logical channels are implemented by incorporating a variable channel id in the packet header. The undesired packets (packets of different channel) are filtered by examining this variable in the received packet header. For the filtering, the changes have been done to Mac 80211 class. Each outgoing packet from MAC has its channel id variable set to appropriate channel. All interfaces like uptarget, downtarget, etc. have been changed such that all the packets that are received either from the Physical Layer or Link Layer are first received by the dual MAC. The determination of channel for an outgoing packet can be either done by changing ARP module or by using a local list of stations on each channel. Currently the second approach is being used. The dual MAC stores the channel-id of the PC in a variable as all the traffic for PCF MAC goes only to the PC. All the packets that are destined for PC are sent on PCF MAC. For incoming packets, the channel-id is determined from the packet header and packet is handed over to appropriate MAC. The corresponding MAC updates its state and after processing the packet and hands it over to link layer.

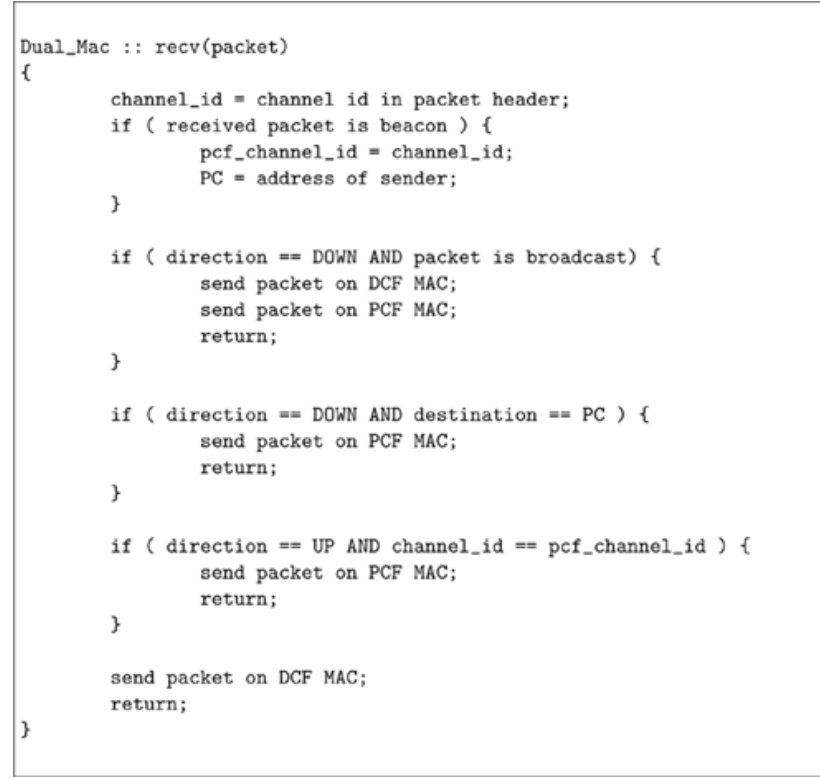

Fig. 2 : Operation of dual MAC 


\section{Simple Scenario and Results}

In order to gain an understating of the dual MAC, we set up a simple scenario as shown in Figure 6. 1. The number of stations in the topology is 18. The receiving stations for all the transmitting stations is the station labeled 0 . The stations numbered 1, 2, 3, 4,5 , and 6 are inner nodes. These stations are within one hop distance of the station 0 . The stations 7,8 , and 9 are the boundary nodes. Rest of the stations in the network are the outer stations. The Figure 7. 2 shows the performance of the dual MAC as compared to the DCF MAC. The number of connections is 15 , and the packet rate is varied to increase the load on the network. It can be observed that as the load on the network increases, the throughput of network with dual nodes becomes better than that of network with DCF nodes only. The graph of packet delivery ratio for with respect to offered load is shown in Figure.

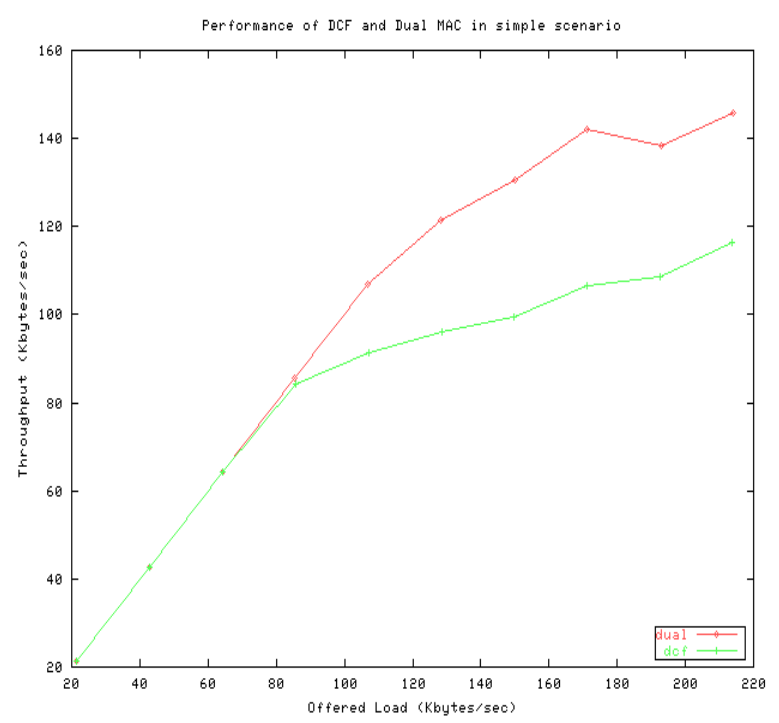

Fig 3 : Packet Delivery Ratio with respect to Offered Load

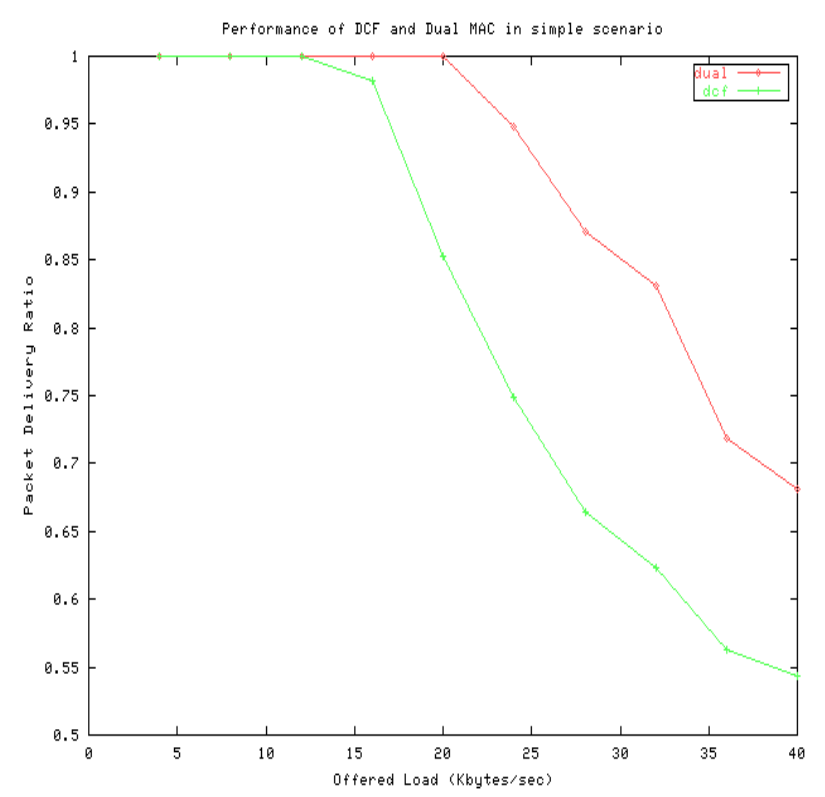

Fig. 4: Packet Delivery Ratio for Dual MAC Vs DCF MAC in simple scenario

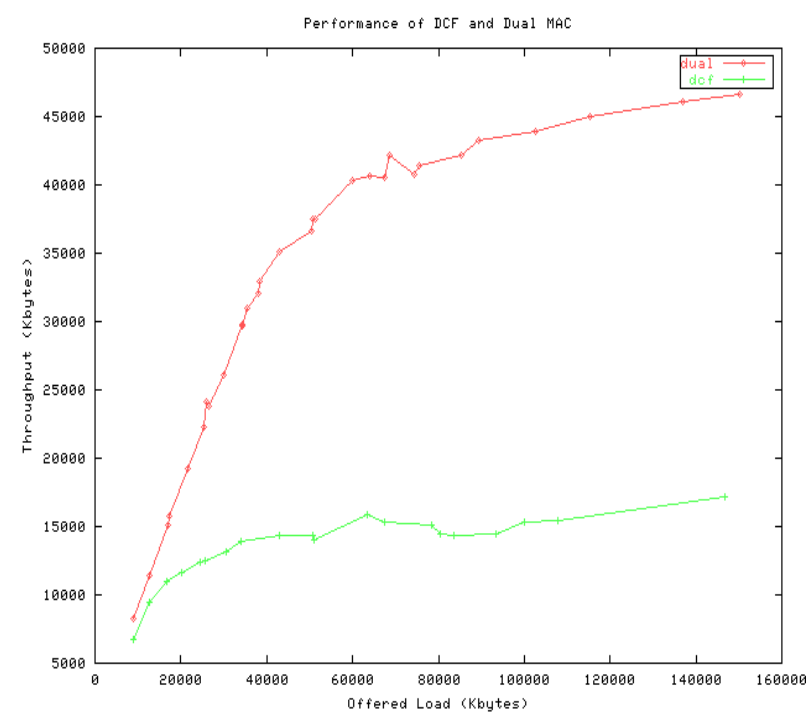

Fig. 5: Dual MAC Vs DCF MAC - overall

As seen from the graphs in the Figure, the performance of dual MAC is considerably better than that of DCF MAC. The increase in the performance is attributed to following reasons:

- The PCF and DCF operate at different channels. This means that there is parallelism in the packet transmissions. This also eliminates the hidden node problem in the centralized scenario. 
- The dual MAC offers parallelism by allowing the transmissions and receptions by DCF MAC even if the NAV of PCF MAC is set during the poll by PC. This eliminates the exposed node problem at boundary nodes which are exposed to the PC when the PC polls the stations.

We can see from the graphs that the throughput performance increase with dual MAC is more than twice that of DCF, which is remarkable considering that only few stations have dual MAC.

\section{Conclusion}

The design of a MAC that meets the demand of a multihop wireless network is great challenge. The restrictions like limited bandwidth, low power, and limited transmission range make this challenge even greater. Further, the hidden and exposed node problems offer even more difficulties by increasing the chance of collision. In this work, we have investigated the usefulness of IEEE 802. 11 MAC protocol using the PCF and DCF mechanisms. We find that without modifications, the PCF and DCF are not very useful in multihop networks. We also find that the high traffic in the central region of a real-life centralized multihop network makes the DCF unsuitable. We investigated the use of PCF in central region to cope up the high traffic requirements in the central region. However, it gave rise to exposed and hidden node problems. The dual MAC was designed to eliminate exposed and hidden node problems in the central region of a centralized multihop network. The thesis discussed the design and architecture of dual MAC along with its implementation in NS.

The results show that the dual MAC performs reasonably better than the DCF access mechanism.

However, the dual MAC requires two physical radios and two separate channels - one bound to PCF and another to DCF. We may however, note that the throughput gain is worth the cost of dual MACs since only few nodes need to be equipped with dual MAC.

\section{REFERENCES}

[1]. Charles E. Perkins et. all, Mobile Ad Hoc Networking Working Group, Ad hoc OnDemand Distance Vector (AODV) Routing, http://www. ietf. org/ internet-drafts/draft-ietfmanet-aodv-12. txt.

[2]. David B. Johnson, David A. Maltz, Yih-Chun $\mathrm{Hu}$, and Jorjeta G. Jetcheva, IETF MANET Working Group, The Dynamic Source Routing Protocol for Mobile Ad Hoc Networks (DSR), http://www. ietf. org/internet-drafts/ draft-ietfmanet-dsr-07. txt.

[3]. IEEE Std. 802. 11, Wireless LAN Media Access Control (MAC) and Physical Layer (PHY) Specifications.

[4]. Brian P. Crowe, I. Widjaja, J. Kim, P. Sakai, IEEE 802. 11 Wireless Local Area

[5]. Andreas Kopsel, Jean-Pierre Ebert, and Adam Wolisz, A Performance Comparision of Point and Distributed Coordination Function of an IEEE 802. 11 WLAN in the presence of RealTime Requirements, Proc. of 7th Intl. Workshop on Mobile Multimedia Communications (MoMuC2000), October 23-26, 2002.

[6]. Shugong Xu, Tarek Saadawi Does IEEE 802. 11 MAC Protocol Work Well in Multihop Wireless Ad Hoc Networks, IEEE Communications Magazine, p. 130-137, June 2001.

[7]. Neeraj Poojary, Srikanth V. Krishnamurthy, and Son Dao, Medium Access Control in a Network of Ad Hoc Mobile Nodes with Heterogeneous Power Capabilities, IEEE International Conference on Communications (ICC 2001), volume 3, p. 872-877, 2001.

[8]. Asis Nasipuri, and Samir R. Das, Multichannel CSMA with Signal Power-Based 
[9]. Channel Selection for Multihop Wireless Networks, Proceedings of the IEEE Fall

[10].The VINT project, NS notes and documentation, editors: Kevin Fall and Kannan

[11].Ad Kamerman and Leo Monteban, WaveLAN-II: A High-Performance Wireless

[12].LAN for the Unlicensed band, 1997.

\section{Cite this article as :}

Amit Kumar, "Qualitative Analysis of dual MAC in Multihop Adhoc Wireless Network", International Journal of Scientific Research in Computer Science, Engineering and Information Technology (IJSRCSEIT), ISSN : 2456-3307, Volume 7 Issue 2, pp. 128-136, March-April 2021. Available at doi : https://doi.org/10.32628/CSEIT217235 Journal URL : https://ijsrcseit.com/CSEIT217235 